TITLE:

\title{
Mode bifurcation of a bouncing dumbbell with chirality
}

$\operatorname{AUTHOR}(\mathrm{S})$ :

Kubo, Yoshitsugu; Inagaki, Shio; Ichikawa, Masatoshi; Yoshikawa, Kenichi

\section{CITATION:}

Kubo, Yoshitsugu ...[et al]. Mode bifurcation of a bouncing dumbbell with chirality. Physical Review E 2015, 91(5): 052905.

ISSUE DATE:

2015-05-08

URL:

http://hdl.handle.net/2433/200911

RIGHT:

(C2015 American Physical Society 


\title{
Mode bifurcation of a bouncing dumbbell with chirality
}

\author{
Yoshitsugu Kubo, ${ }^{1}$ Shio Inagaki, ${ }^{2, *}$ Masatoshi Ichikawa, ${ }^{1, \dagger}$ and Kenichi Yoshikawa ${ }^{3}$ \\ ${ }^{1}$ Department of Physics, Kyoto University, Kyoto, 606-8502, Japan \\ ${ }^{2}$ Department of Physics, Chiba University, Chiba, 263-8522, Japan \\ ${ }^{3}$ Faculty of Life and Medical Sciences, Doshisha University, Kyotanabe, 610-0394, Japan \\ (Received 16 February 2014; revised manuscript received 3 March 2015; published 8 May 2015)
}

\begin{abstract}
We studied the behavior of a dumbbell bouncing upon a sinusoidally vibrating plate. By introducing chiral asymmetry to the geometry of the dumbbell, we observed a cascade of bifurcations with an increase in the vibration amplitude: spinning, orbital, and rolling. In contrast, for an achiral dumbbell, bifurcation is generated by a change from random motion to vectorial inchworm motion. A simple model particle was considered in a numerical simulation that reproduced the essential aspects of the experimental observation. The mode bifurcation from directional motion to random motion is interpreted analytically by a simple mechanical discussion.
\end{abstract}

DOI: 10.1103/PhysRevE.91.052905

PACS number(s): 05.45.-a, 45.40.-f, 05.40.-a

\section{INTRODUCTION}

Spatiotemporal self-organization under nonequilibrium is an intriguing research topic in modern physics. Multiparticle systems of active matter have been studied both experimentally and theoretically [1-4]. In particular, the behavior of a bouncing object has attracted considerable interest as a simple nonlinear system under nonequilibrium conditions [5-13]. In fact, various bifurcations, including the appearance of chaos, have been reported. One of the simplest systems is a bouncing ball on a sinusoidally vibrating plate under the influence of gravity [5-8]. This system has been extensively studied as a classical problem of bifurcation and chaos. For periodic motion, the conditions that correspond to stability and bifurcation can be determined from analytical consideration of a two-dimensional mapping [9]. Over the past decade, the behavior of an anisotropic particle bouncing upon a vibrating plate has been actively studied by experiments and theoretical analysis, as well as by numerical simulation [10-13]. It has been shown that an axisymmetric dimer moves either forward or backward, depending on the initial conditions [10]. For a head-tail asymmetric particle, such as a bolt, directional motion was also observed [11].

In this study, we report how the breaking of chiral symmetry creates a new scenario in the mode bifurcation of a bouncing dumbbell. We introduce chirality by twisting a dumbbell consisting of disks that have a center of mass that deviates from the disk center. We observed two-dimensional motion, such as orbital and spin motion, even though we applied only vertical agitation in the horizontal plane isotropically.

\section{EXPERIMENTS}

Figure 1(a) shows a schematic side view of the dumbbell (left) and the system (right). The reported experiments were performed with a chiral asymmetric dumbbell composed of brass disks with a diameter $d_{A}$ of $10 \mathrm{~mm}$ and a brass rod with a diameter of $2 \mathrm{~mm}$. The length of the $\operatorname{rod} l$ is $13 \mathrm{~mm}$. To introduce chiral asymmetry, we drilled four holes

\footnotetext{
*Corresponding author: inagaki@ phys.kyushu-u.ac.jp

${ }^{\dagger}$ Corresponding author: ichi@scphys.kyoto-u.ac.jp
}

with a diameter $d_{B}$ of $4 \mathrm{~mm}$ in each disk at an angular interval $\alpha$ of $0.25 \pi$. Next, we rotated the front disk clockwise with respect to the rear one by the angle $\beta$ and fixed on this geometry. The aluminum plate was placed horizontally and then vibrated vertically with $z(t)=A \sin (2 \pi f t)$ by an electromagnetic shaker. The oscillation frequency $f$ and the dimensionless acceleration $\Gamma=4 \pi^{2} f^{2} A / g$, where $A$ is the amplitude of the plate oscillation and $g$ is the gravitational acceleration, were adopted as independent control parameters. The conventional restitution coefficient $e$ measured with a collision of a brass sphere on the aluminum are $0.38 \pm 0.02$.

Figures 1(b)-1(d) show the characteristic modes of the spontaneous motions of the dumbbell. The twist angle $\beta$ and $f$ are set to $0.75 \pi$ and $50 \mathrm{~Hz}$, respectively. In Figs. 1(b) and 1(c), the left panels show a series of snapshots with a time interval of $1 \mathrm{~s}$. The right panels show the trajectories of the dumbbell center and the orientation of the dumbbell. For $\Gamma<1$, the dumbbell may remain on the plate. Depending on the dimensionless acceleration, for $\Gamma>1$, three distinctive modes were observed. When $\Gamma$ is slightly above unity, the dumbbell itself spins, and the centroid moves randomly [spin + random (SR mode), Fig. 1(b), $(\Gamma=1.3)$ ]. When $\Gamma$ increases to 1.4 , the dumbbell motion bifurcates into a distinctive directional motion accompanied by spinning with an identical period [orbital (O) mode, Fig. 1(c)]. When we set $\Gamma$ even higher, as shown in Fig. 1(d) $(\Gamma=2.5)$, the dumbbell rolled along the axis of the rod (rolling mode). See Supplemental Material [14] for movies.

Figure 2 shows the dependence of the angular velocity $\Omega$ on the twist angle $\beta$ for the SR mode ( $\Gamma=1.3$ ). When $\beta=0$, the dumbbell does not spin. For $\beta>0$, the dumbbell spins clockwise $(\Omega<0)$, and otherwise it spins counterclockwise $(\Omega>0)$. The direction of rotation is determined so that the heavier half of the disks at the ends of the dumbbell is in the front. For example, in the sketch on the left in Fig. 1(a), the left half of the front disk is heavier than the right half for $\beta>0$. Thus, the dumbbell rotates so that the front disk moves from right to left, and the dumbbell rotates clockwise. This behavior is the same as that of a rattleback, a popular science toy that rotates in a preferred direction because of its chirality. This indicates that the introduction of chirality into the geometry of the dumbbell causes spinning motion. It was also found 
(a)

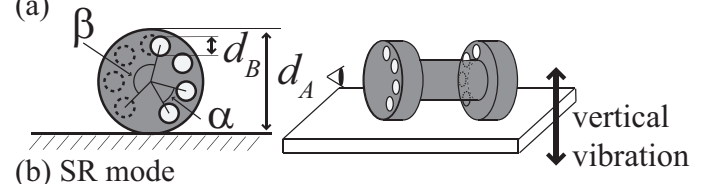

(b) SR mode

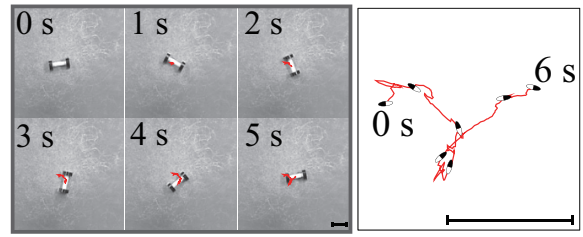

(c) $\mathrm{O}$ mode

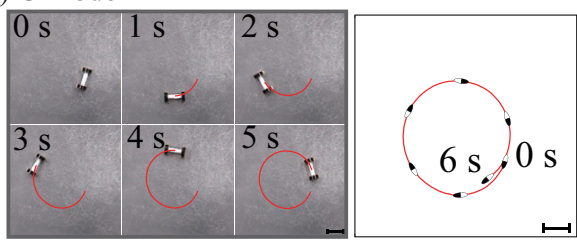

(d) Rolling mode

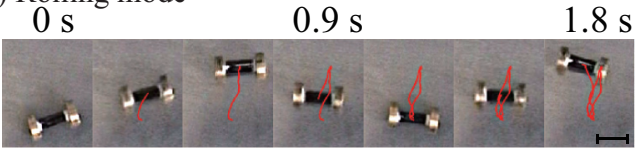

FIG. 1. (Color online) (a) Experimental setup. Left: side view of a chiral asymmetric dumbbell. Right: overall setup. $\alpha$ and $\beta$ are chirality parameters. (b-d) Examples of the characteristic spontaneous motion of a chiral asymmetric dumbbell. The vibration frequency of the plate was fixed at $50 \mathrm{~Hz}$. The scale bar is $10 \mathrm{~mm}$. (b, c) Left panels: Series of snapshots with a time interval of $1 \mathrm{~s}$. Right panels: Trajectories of the center of the dumbbell. (b) Spin + random $(\mathrm{SR})$ mode $(\Gamma=1.3)$ : the dumbbell itself spins, and the centroid moves randomly. (c) Orbital $(\mathrm{O})$ mode $(\Gamma=1.4)$ : orbital motion is accompanied by spinning; the direction of the orbital motion is determined stochastically. (d) Rolling mode $(\Gamma=2.5)$ : series of snapshots with a time interval of $0.3 \mathrm{~s}$. The dumbbell rolls along the axis of the rod with rhythmic switching of the rolling direction.

that $|\Omega|$ decreases with increasing the length of the rod $l$. When $\Omega(\beta)$ was normalized by the maximum values $\Omega_{\max }$, the angular velocities for different rod lengths converged to an identical curve, as shown in the inset of Fig. 2.

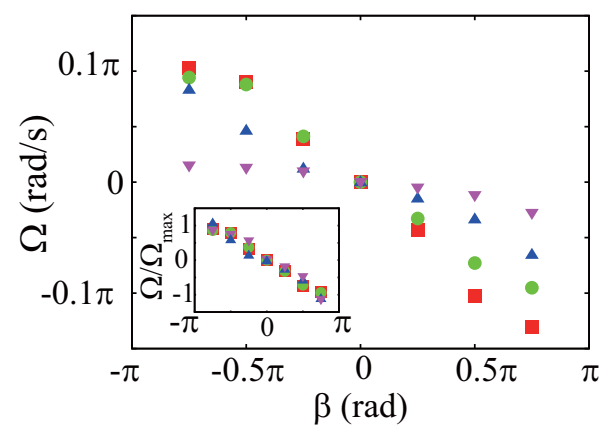

FIG. 2. (Color online) Angular velocity of spin motion $\Omega$ in the SR mode $(\Gamma=1.3)$ as a function of $\beta$ for different rod lengths $l: 12.5$ (squares), 17.5 (circles), 22.5 (triangles), and 27.5 (inverted triangles) $\mathrm{cm}$. Inset: $\Omega$ normalized to the maximum angular velocity $\Omega_{\max }$ of the spin motion as a function of $\beta$. (a)

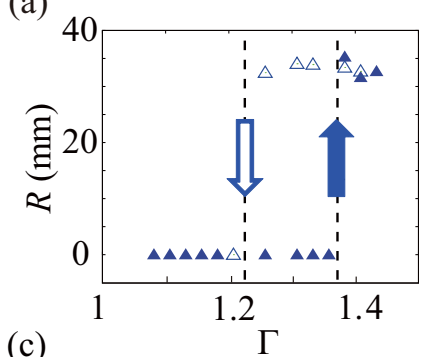

(b)

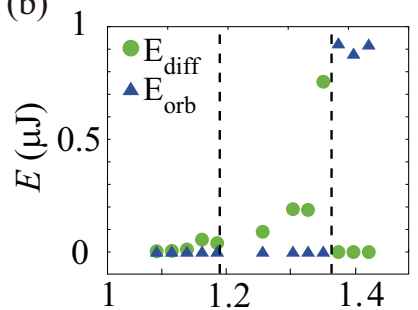

(c)

(d)

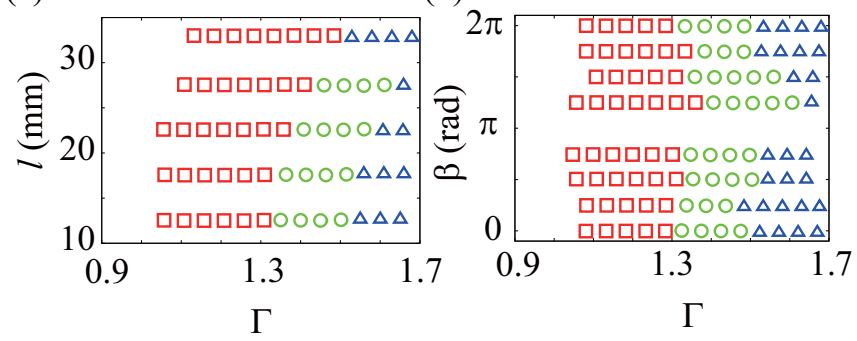

FIG. 3. (Color online) (a) Orbital radius. An increase (decrease) in $\Gamma$ is shown by filled (open) triangles. (b) Kinetic energy of the dumbbell as a function of $\Gamma$. (c) and (d) Phase diagrams of the dumbbell motion: SR mode $(\square)$, O mode $(\bigcirc)$, and Rolling mode $(\triangle)$. (c): $\Gamma$ vs. the length of the $\operatorname{rod} l$, and (d): $\Gamma$ vs. the twist angle $\beta$.

To quantitatively evaluate the modes of dumbbell motion, we calculated the mean square displacement as a function of time for various $\Gamma$ values at a fixed frequency $f=50 \mathrm{~Hz}$. We performed fitting using the function

$$
\left\langle x(t)^{2}\right\rangle=2 R^{2}(1-\cos \dot{\phi} t)+v^{2} t^{2}+D t,
$$

where $R$ is the orbital radius, $\dot{\phi}$ is the angular velocity of orbital motion, $v$ is the velocity of ballistic motion, and $D$ is the diffusion coefficient. The three terms on the right-hand side correspond to orbital, ballistic, and diffusive motion, respectively. In the $\mathrm{SR}$ and $\mathrm{O}$ modes, the velocity of ballistic motion was negligible. In Fig. 3(a), the orbital radius $R$ is plotted as a function of $\Gamma$. An increase (decrease) in $\Gamma$ is shown by filled (open) triangles. As $\Gamma$ increases to 1.4 , the orbital radius in the $\mathrm{SR}$ mode is approximately zero. At $\Gamma=1.4$, the dumbbell motion switches to the $\mathrm{O}$ mode and the orbital radius becomes finite. For $\Gamma>1.4$, the orbital radius appears to be independent of $\Gamma$. With decreasing $\Gamma$, orbital motion persists even for $\Gamma<1.4$, and the motion switches back to the SR mode at $\Gamma=1.2$. The SR and $\mathrm{O}$ modes switch in a subcritical manner. Next, we estimated the kinetic energy to investigate energy conversion near the bifurcation point. The kinetic energies of the random and orbital motions, $E_{\text {diff }}$ and $E_{\text {orb }}$, respectively, were estimated as

$$
\begin{gathered}
E_{\mathrm{diff}}=\frac{1}{2} m \bar{v}^{2}=\frac{1}{2} m \frac{4 D}{\delta t}, \\
E_{\mathrm{orb}}=\frac{1}{2} m(R \dot{\phi})^{2},
\end{gathered}
$$

where $\delta t$ is the time interval of the collisions (approximately $1 / f)$. In Fig. 3(b), $E_{\text {diff }}$ and $E_{\text {orb }}$ are plotted as functions of $\Gamma$. $E_{\text {diff increases monotonically and then collapses at the }}$ bifurcation point. In contrast, $E_{\text {orb }}$ is approximately zero until it becomes finite at that point. Thus, it becomes evident that, 
(a)

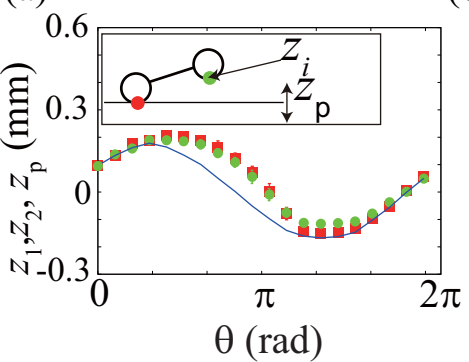

(b)

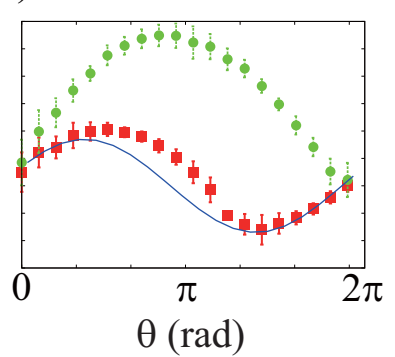

FIG. 4. (Color online) Experimental results for the vertical trajectory of the tips of the dumbbell as a function of the vibration phase. The height of the plate is shown as $z_{p}(\theta)$ by the solid blue line. The heights of the lowest points of the disks are shown as $z_{1}(\theta)$ and $z_{2}(\theta)$ by circles (green) and squares (red), respectively. (a) SR mode and (b) O mode.

in conjunction with mode bifurcation, the energy of random fluctuation is converted to that of orbital motion.

Figures 3(c) and 3(d) show phase diagrams of the dumbbell motion, which were plotted for increasing $\Gamma$ at a fixed rod length $l$ and twist angle $\beta$, respectively. For different rod lengths $l$ and twist angles $\beta$, the SR mode appears at lower $\Gamma$, and the rolling mode appears at higher $\Gamma$. Between them, the $\mathrm{O}$ mode is observed. As shown in Fig. 3(c), the critical acceleration at which the mode bifurcates from the SR mode to the $\mathrm{O}$ mode increases as $l$ increases. We also confirmed that the bifurcation point is nearly independent of the frequency. In addition, our experiment shows that the bifurcation point remains almost constant with a change in the twist angle $\beta$, as shown in Fig. 3(d). These results indicate that the chirality has a negligible effect on the magnitude of $\Gamma$ at the bifurcation from the SR mode to the $\mathrm{O}$ mode.

To evaluate the difference in vertical trajectory between the SR and $\mathrm{O}$ modes, lateral images were captured by a high-speed camera with a spatial precision of ca. $60 \mu \mathrm{m}$. Figure 4 shows a plot of the vertical coordinates of the plate and the lowest points of the disks as a function of the vibration phase $\theta$. In the SR mode, the two disks jump up when the acceleration of the plate is smaller than $-g$, and then land on the plate at different phases [Fig. 4(a)]. In contrast, in the O mode, one of the disks of the dumbbell hits the plate at an identical phase, whereas the other appears to stay near the plate throughout the cycle [Fig. 4(b)]. This head-tail asymmetric mode is the origin of the directional motion of the dumbbell, as pointed out in a previous study on an axisymmetric dimer [10].

\section{NUMERICAL SIMULATION}

To understand the scenario in which spinning motion occurs, we considered a simple model particle with chiral asymmetry. The model particle consists of two spheres connected by a weightless rigid bond. When there is no chiral asymmetry, as shown in Fig. 5(i), the spheres consist of two hemispheres that have different mass densities, $\rho_{1}$ (blue) for $y \geqslant 0$ and $\rho_{2}$ (green) for $y<0$. Chiral asymmetry is introduced by twisting the lower sphere with respect to the upper sphere by the angle $\beta^{\prime}$ [Fig. 5(ii)]. The equation of motion for the center of the model particle (the midpoint of

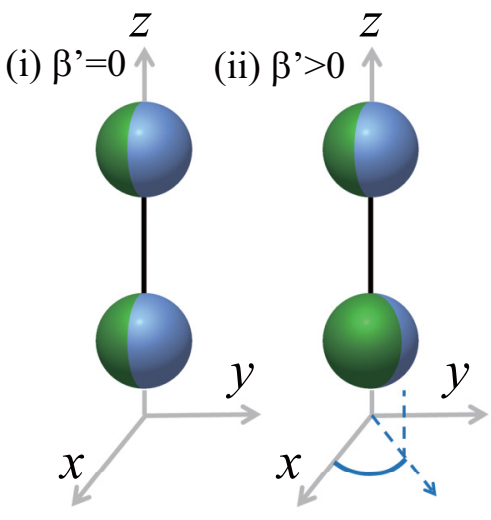

FIG. 5. (Color online) Schematic illustration of the chiral symmetric model particle. The two hemispheres have different mass densities: $\rho_{1}$ (blue) and $\rho_{2}$ (green), where $\rho_{1}>\rho_{2}$.

the bond) $\boldsymbol{x}$ and the angular velocity $\boldsymbol{\omega}$ are written as

$$
M \frac{d^{2} \boldsymbol{x}}{d t^{2}}=\sum_{i=1}^{2} \boldsymbol{F}_{i}-M \boldsymbol{g}, \quad \frac{d}{d t}(\boldsymbol{I} \boldsymbol{\omega})=\sum_{i=1}^{2} \boldsymbol{r}_{i} \times \boldsymbol{F}_{i}-\eta_{r} \boldsymbol{\omega}
$$

where $M$ is the mass of the model particle, $\boldsymbol{g}$ is the gravitational acceleration, $\boldsymbol{I}$ is the inertia tensor, $\boldsymbol{F}_{i}$ is the contact force applied by the bottom to sphere $i$, and $\boldsymbol{r}_{i}=\boldsymbol{x}_{c, i}-\boldsymbol{x}$ is the radius vector from $\boldsymbol{x}$ to the contact point of sphere $i$ on the plate $\boldsymbol{x}_{c, i}$. In the equation for rotation, the second term on the right-hand side represents the rolling resistance [15]. The contact force $\boldsymbol{F}_{i}$ and the rolling resistance are applied only when sphere $i$ is in contact with the vibrating plate. Because there are two spheres at the ends of the particle, the index $i$ ranges from 1 to 2 . The force law between sphere $i$ and the plate is

$$
\boldsymbol{F}_{n, i}=k_{n} \delta_{n}^{3 / 2} \boldsymbol{n}-\eta_{n} \dot{\delta}_{n} \delta_{n}^{1 / 2} \boldsymbol{n}, \quad \boldsymbol{F}_{t, i}=k_{t} \delta_{t} \boldsymbol{t}-\eta_{t} \dot{\delta}_{t} \boldsymbol{t},
$$

where $\boldsymbol{F}_{n, i}$ and $\boldsymbol{F}_{t, i}$ are normal and tangential interactions, respectively; $\delta_{n}=z_{p}-\left(z_{i}-R\right)$ is the normal component of the overlap between the sphere and the plate; $z_{p}$ and $z_{i}$ are the $z$ coordinates of the plate and sphere $i$, respectively; $R$ is the radius of the spheres. Further, $\dot{\delta_{n}}=\boldsymbol{v}_{c} \cdot \boldsymbol{n}$ is the time derivative of $\delta_{n}$, where $\boldsymbol{v}_{c}=\boldsymbol{v}_{i}-\boldsymbol{v}_{p}$ is the relative velocity, and $\boldsymbol{v}_{i}$ and $\boldsymbol{v}_{p}$ are the velocities of sphere $i$ and the bottom plate, respectively. Note that the force law consists of the Hertzian elastic force and viscous damping [16]. Because the contact on the plate is always in the vertical direction, the normal unit vector $\boldsymbol{n}$ is $\boldsymbol{e}_{z}$, whereas the tangential vector is defined as $\boldsymbol{t}=\boldsymbol{e}_{x}+\boldsymbol{e}_{y}$. The tangential component of the relative velocity of the contact point is defined as $\dot{\delta}_{t}=\left(\boldsymbol{v}_{c}-R \boldsymbol{\omega} \times \boldsymbol{n}\right) \cdot \boldsymbol{t}$. When the sphere starts to touch the plate at $t=t_{0}$, it puts a "virtual" spring at the contact point, and $\delta_{t}(t)=-\int_{t_{0}}^{t} \dot{\delta}_{t}\left(t^{\prime}\right) d t^{\prime}$ is the total tangential displacement at time $t$. The parameter values we used are $R=1 \mathrm{~cm}, k_{n}=2.0 \times 10^{12} \mathrm{~g} /\left(\mathrm{cm}^{1 / 2} \mathrm{~s}^{2}\right)$, $k_{t}=1.14 \times 10^{12} \mathrm{~g} / \mathrm{s}^{2}, \eta_{n}=5.32 \times 10^{7} \mathrm{~g} /\left(\mathrm{cm}^{1 / 2} \mathrm{~s}\right)$, and $\eta_{t}=$ $3.04 \times 10^{7} \mathrm{~g} / \mathrm{s}$. Note that the normal restitution coefficient is approximately 0.4 . The mass densities of the hemispheres of the particle are $\rho_{1}=8.85$ and $\rho_{2}=6.85 \mathrm{~g} / \mathrm{cm}^{3}$. The parameters are chosen to fit the material properties of the model 
(a)

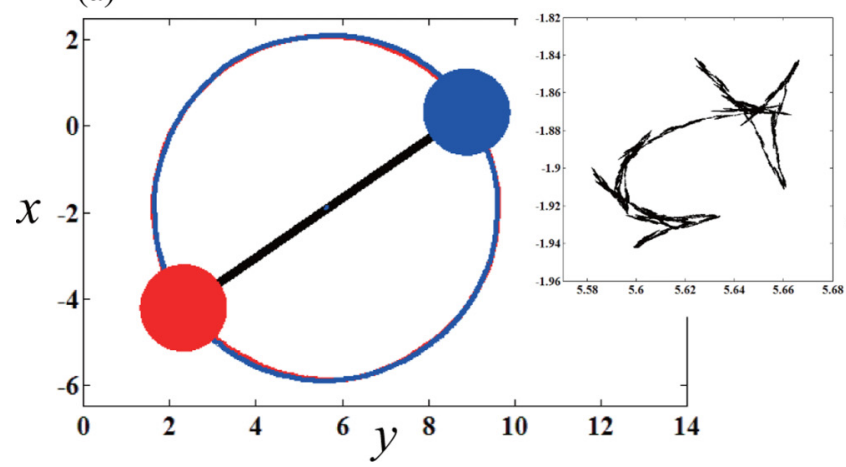

(b)

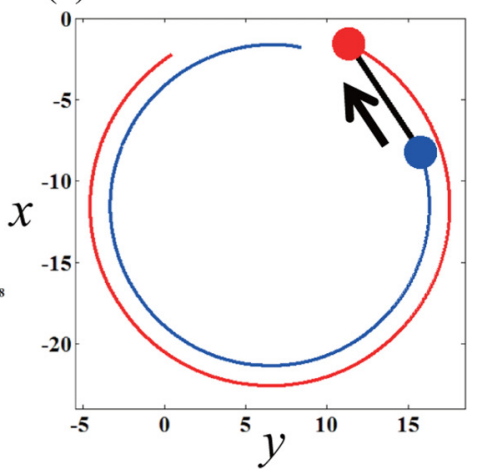

FIG. 6. (Color online) Horizontal trajectories of the tips of the dumbbell at $f=40 \mathrm{~Hz}, l=8 \mathrm{~cm}$, and $\beta^{\prime}=0.01 \pi$. (a) SR mode at $\Gamma=1.68$ for $2.7 \times 10^{4} \mathrm{~s}$ (inset: trajectory of the center of the dumbbell) and (b) $\mathrm{O}$ mode at $\Gamma=1.74$ for $2 \times 10^{3} \mathrm{~s}$.

particle to those of the dumbbell. We solved the equations of motion of the center position of the model particle by employing the Adams method with a time step of $5.0 \times 10^{-7} \mathrm{~s}$. Conversely, the angular velocity was obtained by calculating the inertia tensor from its quaternion at each time step in the laboratory frame. In the initial configuration, as shown in Fig. 5(ii), the moment of the inertia tensor $\boldsymbol{I}^{0}$ is obtained analytically as

$$
\begin{gathered}
I_{x x}^{0}=\frac{4}{5} m R^{2}+\frac{1}{2} m l^{2}-m y_{g}^{2}, \\
I_{y y}^{0}=\frac{4}{5} m R^{2}+\frac{1}{2} m l^{2}-m x_{g}^{2}, \\
I_{z z}^{0}=\frac{4}{5} m R^{2}-m\left(x_{g}^{2}+y_{g}^{2}\right), \\
I_{x z}^{0}=-\frac{\pi}{8}\left(\rho_{1}-\rho_{2}\right) \sin \beta^{\prime} R^{4} l, \\
I_{y z}^{0}=-\frac{\pi}{8}\left(\rho_{1}-\rho_{2}\right)\left(1-\cos \beta^{\prime}\right) R^{4} l, \\
I_{x y}^{0}=m x_{g} y_{g}, \quad I_{y x}^{0}=I_{z x}^{0}=I_{z y}^{0}=0,
\end{gathered}
$$

where $\quad x_{g}=\sin \beta^{\prime} \frac{\pi R^{4}}{8 M}\left(\rho_{1}-\rho_{2}\right) \quad$ and $\quad y_{g}=-(1+$ $\left.\cos \beta^{\prime}\right) \frac{\pi R^{4}}{8 M}\left(\rho_{1}-\rho_{2}\right)$. The mass of a sphere is defined as $m=\frac{2}{3} \pi\left(\rho_{1}+\rho_{2}\right) R^{3}$. We established the simulations in the upright position on the plate by applying the angular velocity $\omega_{x}$ and $\omega_{y}$. Figures 6 and 7 show the qualitative (a)

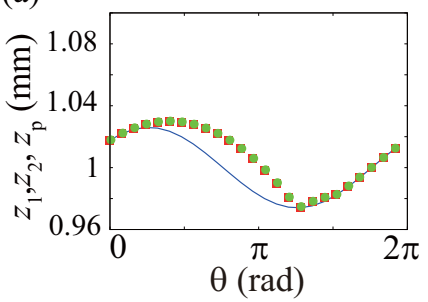

(b)

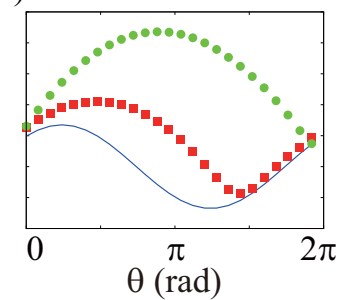

FIG. 7. (Color online) Vertical trajectories of the tips of the dumbbell as a function of the vibration phase at $f=40 \mathrm{~Hz}, l=8$ $\mathrm{cm}$, and $\beta^{\prime}=0.01 \pi$. (a) SR mode at $\Gamma=1.68$ and (b) $\mathrm{O}$ mode at $\Gamma=1.74$. reproduction of the horizontal and vertical trajectories of the tips of the model particle, respectively. The typical time scale of the dumbbell motion in the numerical simulation is about $10^{3}$ times larger than that in the experimental results. We conducted numerical simulations with a symmetric dumbbell $\left(\rho_{1}=\rho_{2}\right)$ and measured the translational velocity for different stiffnesses. The viscous coefficient $\eta_{n}$ was chosen so that the restitution coefficient is identical. As shown in Fig. 8, the translational velocity depends on the stiffness parameter $k_{n}$. In addition, we found that the translational velocity is an increasing function of the contact duration (inset of Fig. 8). Consequently, it makes the translational motion faster. In the experiments, the contact duration is expected to be even longer for a linear contact because of the discoid shape of the ends of the dumbbell. Because we chose the stiffness to fit the material properties of the dumbbell particle, the translational velocity of the dumbbell appeared to be much smaller than that observed experimentally. Note that the essential aspect of the dumbbell motion was reproduced using the simple model particle.

The dependence of the angular velocity $\Omega$ on the twisting parameter $\beta^{\prime}$ is shown in Fig. 9. $|\Omega|$ tends to increase when the bond length $l$ of the particle shortens. This result is consistent with the experimental result (Fig. 2). In addition,

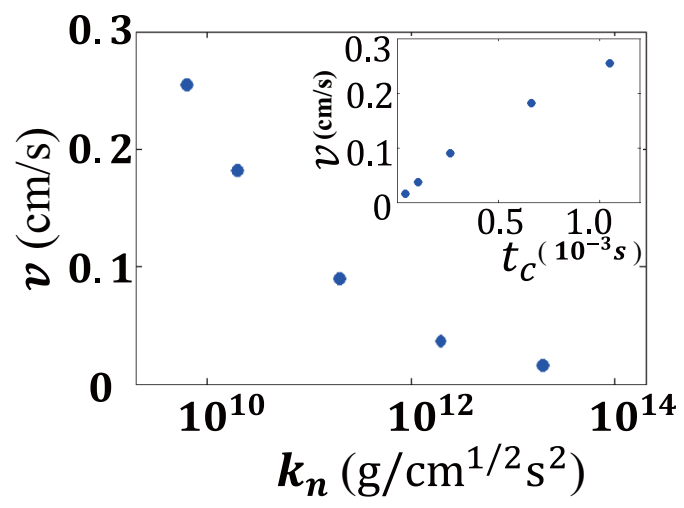

FIG. 8. (Color online) Dependence of translational velocity of a symmetric dumbbell on the stiffness $k_{n}$. The viscous coefficient is chosen so that the restitution coefficient is identical. Inset: the relation between translational velocity $v$ and contact duration $t_{c}$ for different stiffnesses. 


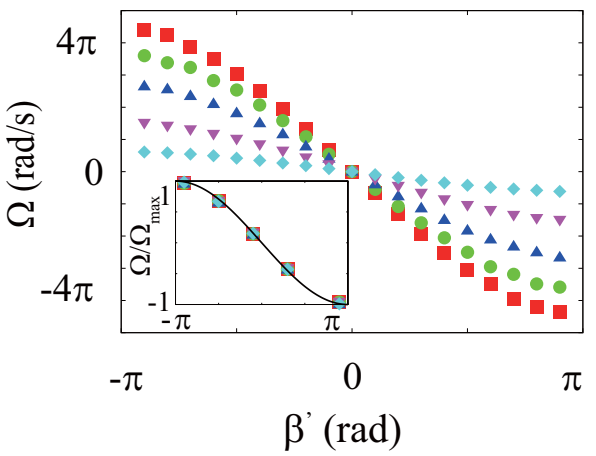

FIG. 9. (Color online) Angular velocity of the spin motion $\Omega$ as a function of $\beta^{\prime}$ for different rod lengths $l: 3.5$ (squares), 4 (circles), 5 (triangles), 6 (inverted triangles), 8 (diamonds) $\mathrm{cm}$. Inset: scaled by the maximum value.

when we normalized the curves by their maximum values, they converged to the identical curve fitted as $\sin \left(\beta^{\prime} / 2\right)$, as shown in the inset in Fig. 9. Our theoretical understanding of the spinning motion should be deepened by clarifying the origin of $\sin \left(\beta^{\prime} / 2\right)$.

Starting from the $\mathrm{O}$ mode with a decrease in $\Gamma$, the mode bifurcates into the SR mode at $\Gamma_{c}$. This $\Gamma_{c}$ is the lower limit at which the upper sphere can maintain periodic collision. We examined the dependence of the bifurcation point $\Gamma_{c}$ on the restitution coefficient as shown in Fig. 10. The restitution coefficient is defined by the ratio of the relative preimpact velocity of the upper sphere against the bottom plate to the relative postimpact velocity as

$$
V-\dot{z}_{p}=e^{\prime}\left(U-\dot{z}_{p}\right)
$$

where $V$ and $U$ are the preimpact and postimpact velocities of the upper sphere, respectively, and $\dot{z}_{p}$ is the velocity of the bottom plate at collision. Note that this reduced restitution coefficient $e^{\prime}$ is not equivalent to an ordinary restitution coefficient $e$ defined with a normal two-body collision. We will discuss this point in detail in Sec. IV. The curves for the

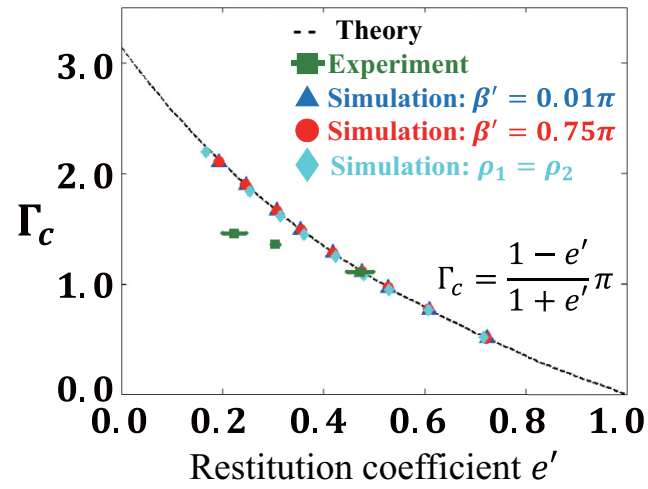

FIG. 10. (Color online) Dependence of the critical acceleration $\Gamma_{c}$ on the restitution coefficient $e^{\prime}$. Lower limit of steady collision in the theoretical analysis (dotted line), experiments (green square), and numerical simulation with $\beta^{\prime}=0.01 \pi$ (blue triangles), $\beta^{\prime}=$ $0.75 \pi$ (red circles), and $\rho_{1}=\rho_{2}$ (cyan diamonds). Experimental data are given for rubber $\left(e^{\prime}=0.223 \pm 0.024\right)$, aluminum $\left(e^{\prime}=\right.$ $0.305 \pm 0.008)$, and glass plates $\left(e^{\prime}=0.472 \pm 0.026\right)$. twist angle $\beta^{\prime}=0.01 \pi$ and $0.75 \pi$ are shown to be identical. A numerical simulation with a symmetric dumbbell $\left(\rho_{1}=\rho_{2}\right)$ was also conducted, and $\Gamma_{c}$ appeared to be on the same curve as those of the chiral particles. Thus, the twist angle $\beta^{\prime}$ is shown to have no effect on $\Gamma_{c}$. To examine the dependence of $\Gamma_{c}$ on the restitution coefficient experimentally, we also conducted experiments using different substrates, such as glass and rubber. The conventional restitution coefficients $e$ measured with a collision of a brass sphere on the glass and rubber plates are $0.54 \pm 0.04$ and $0.48 \pm 0.04$, respectively. As shown in Fig. 10, for the glass plate $\left(e^{\prime}=0.472 \pm 0.026\right)$, the experimental value of $\Gamma_{c}$ in the experiments appears to agree with the numerical results. With a decrease in $e^{\prime}$, the experimental $\Gamma_{c}$ deviates from the numerical results. In the next section, we will analytically obtain $\Gamma_{c}$ with a simple mechanical model. In addition, we will discuss causes of the deviation.

\section{STABILITY ANALYSIS OF THE DIRECTIONAL MOTION}

In this section, we aim to obtain the critical acceleration $\Gamma_{c}$ as a function of the restitution coefficient and compare it with the experimental and numerical results shown in Fig. 10. As indicated in Fig. 3(d), the bifurcation between the SR and $\mathrm{O}$ modes is expected to be independent of the chirality. At $\Gamma_{c}$, the dumbbell motion switches from directional to random behavior. Therefore, for simplicity, we focus on the bifurcation from directional to random motion of a symmetric dumbbell. Here, we consider a symmetric dimer $\left(\rho_{1}=\rho_{2}\right)$ placed parallel to the $x$ axis, as shown in Fig. 11. Because of its symmetry, the motion of the dimer will be restricted to the $x-z$ plane. The orientation of the rod on the $x-z$ plane is defined as $\xi$. The time derivative of $\xi$ corresponds to $-\boldsymbol{\omega}_{y}$. As shown in Eq. (5), the equations of motion are written as

$$
\frac{d}{d t}(\boldsymbol{I} \boldsymbol{\omega})=\sum_{i=1}^{2} \boldsymbol{N}_{i}=\sum_{i=1}^{2} \boldsymbol{r}_{i} \times \boldsymbol{F}_{i},
$$

where $N$ is the torque. The radius vectors are written as

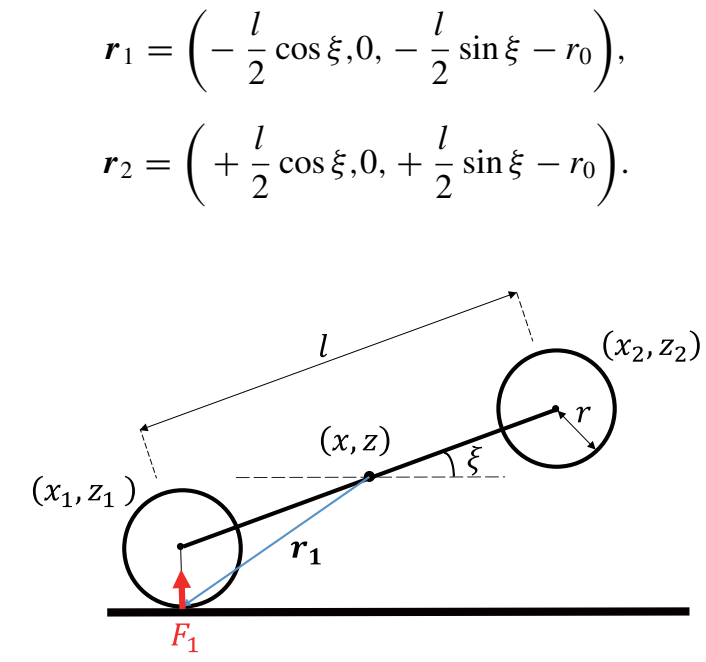

FIG. 11. (Color online) Schematic drawing of bouncing dimer model. 
From the numerical simulation, we confirmed that $\Gamma_{c}$ shifts only $1 \%$ at maximum by switching off the tangential contact force. Thus, we consider that the tangential friction is not crucial for the stability of the $\mathrm{O}$ mode and take into account only the normal force in this model. The normal force applied by the bottom plate is defined as

$$
\boldsymbol{F}_{1}=\left(0,0, F_{1}\right), \quad \boldsymbol{F}_{2}=\left(0,0, F_{2}\right) .
$$

$F_{1}$ and $F_{2}$ are nonzero only when the dimer ends are in contact with the bottom plate. For sphere 1, the condition of contact is written as

$$
z-\left(\frac{l}{2} \sin \xi+r_{0}\right) \leqslant z_{p},
$$

where $z$ and $z_{p}$ are the $z$ coordinates of the center of mass of the dimer and the position of the bottom plate, respectively. Similarly, for sphere 2 , the condition of contact is

$$
z-\left(-\frac{l}{2} \sin \xi+r_{0}\right) \leqslant z_{p} .
$$

The normal forces are

$$
\begin{gathered}
\boldsymbol{N}_{1}=\boldsymbol{r}_{1} \times \boldsymbol{F}_{1}=\left(0, \frac{l}{2} F_{1} \cos \xi, 0\right), \\
\boldsymbol{N}_{2}=\boldsymbol{r}_{2} \times \boldsymbol{F}_{2}=\left(0,-\frac{l}{2} F_{2} \cos \xi, 0\right) .
\end{gathered}
$$

For a symmetric dimer, the asymmetric components of $\boldsymbol{I}$ are zero. Because the motion of the dimer is now restricted to the $x-z$ plane, the inertia tensor $I_{y y}$ is time independent. Therefore, the time evolution equation for $\xi$ is

$$
-I \ddot{\xi}=N_{1}+N_{2}=\frac{l}{2} \cos \xi\left(F_{1}-F_{2}\right),
$$

where $I$ is $I_{y y}=(4 / 5) m R^{2}+(1 / 2) m l^{2}$. The vertical velocities of spheres 1 and 2 are written as

$$
\dot{z_{1}}=-\frac{l}{2} \dot{\xi} \cos \xi+\dot{z}, \quad \dot{z_{2}}=+\frac{l}{2} \dot{\xi} \cos \xi+\dot{z}
$$

When the orientation of the dimer is sufficiently small, it can be assumed that $\xi \ll 1, \cos \xi \sim 1$, and $\sin \xi \sim \xi$. Then Eqs. (21) and (22) are simplified as

$$
\begin{gathered}
I \ddot{\xi}=\frac{l}{2}\left(-F_{1}+F_{2}\right), \\
\dot{z_{1}}=-\frac{l}{2} \dot{\xi}+\dot{z}, \quad \dot{z_{2}}=+\frac{l}{2} \dot{\xi}+\dot{z} .
\end{gathered}
$$

The equation of motion of the center of mass $z$ is written as

$$
M \ddot{z}=-M g+F_{1}+F_{2},
$$

where $M=2 m$ is the total mass of the dimer. By taking the time derivative of Eq. (24) and substituting Eqs. (23) and (25), we obtain

$$
\begin{aligned}
& \ddot{z}_{1}=-g+\left(\frac{1}{M}+\frac{l^{2}}{4 I}\right) F_{1}+\left(\frac{1}{M}-\frac{l^{2}}{4 I}\right) F_{2}, \\
& \ddot{z}_{2}=-g+\left(\frac{1}{M}-\frac{l^{2}}{4 I}\right) F_{1}+\left(\frac{1}{M}+\frac{l^{2}}{4 I}\right) F_{2} .
\end{aligned}
$$

Here, we rewrite $z_{1}-r_{0}$ and $z_{2}-r_{0}$ as $z_{1}$ and $z_{2}$, respectively. These equations can be written as

$$
\begin{aligned}
& \ddot{z}_{1}=-g+(1-\alpha) F_{1}^{\prime}+\alpha F_{2}^{\prime}, \\
& \ddot{z}_{2}=-g+\alpha F_{1}^{\prime}+(1-\alpha) F_{2}^{\prime},
\end{aligned}
$$

where $F_{1}^{\prime}=F_{1} / m$ and $F_{2}^{\prime}=F_{2} / m$. The coefficient $\alpha$, which characterizes the cross-coupling interaction, is written as

$$
\alpha=\frac{4(r / l)^{2}}{5+8(r / l)^{2}} .
$$

This reflects the effect of the shape of the dumbbell on the equations of motion. The conditions of contact are $z_{1} \leqslant z_{p}$ and $z_{2} \leqslant z_{p}$. Now we obtain the equations of motion of the spheres with cross-coupling terms. When $\alpha$ is sufficiently small, the motions of the spheres at the ends of the dimer can be described as those of two bouncing balls under gravity weakly interacting with each other.

To obtain the lower limit of $\Gamma$ for the directional motion, we consider asymmetric collisions. Suppose sphere 1 is on the lower end and stays on the bottom plate throughout the oscillation phase, $z_{1}=z_{p}$. Then the normal force applied to sphere 1 by the bottom plate is written from Eq. (28) as

$$
F_{1}^{\prime}=\left(g+\ddot{z}_{p}\right) /(1-\alpha) .
$$

Here we neglect the impulsive force $\alpha F_{2}^{\prime}$ resulting from the collision of sphere 2 . The effect of bouncing of sphere 1 due to $\alpha F_{2}^{\prime}$ will be discussed in the Appendix. For $\Gamma>1$, sphere 1 may detach from the bottom plate when $A \omega^{2} \sin \left(\omega t_{d}\right)=g$. The effect of the flight and collision of sphere 1 will also be discussed in the Appendix. The upper sphere, sphere 2, collides with the bottom plate periodically. We assume that the duration of contact with the bottom is sufficiently short (typically $10^{-4} \mathrm{~s}$ in the numerical simulation). In addition, the contact force from the bottom plate is assumed to be sufficiently large compared with the gravitational force. Thus, during a collision, we neglect the displacement of the bottom plate, gravity $(g)$, and the normal contact force $F_{1}$ of sphere 1 . Then, the time evolution equation of sphere 2 during a collision can be written as

$$
m \ddot{\delta} \ddot{z}_{2}=(1-\alpha)\left(-k_{n}\left|\delta z_{2}\right|^{\frac{3}{2}}-\eta_{n}\left|\delta z_{2}\right|^{\frac{1}{2}} \delta \dot{z}_{2}\right),
$$

where $\delta z_{2}=z_{2}-z_{p}$. From the time integral of Eq. (32), we can calculate the restitution coefficient of sphere 2 as

$$
V_{i}-\dot{z}_{p}\left(t_{i}\right)=e^{\prime}\left[U_{i}-\dot{z}_{p}\left(t_{i}\right)\right],
$$

where $V_{i}$ and $U_{i}$ are the preimpact velocity and postimpact velocity, respectively, of the $i$ th collision at time $t_{i}$. The restitution coefficient $e^{\prime}$ of sphere 2 is a function of $k_{n}, \eta_{n}$, and $\alpha$. Note that in the limit of $\alpha=0, e^{\prime}$ is identical to the ordinary restitution coefficient of a single bouncing ball $e$. For a linear spring model, $F_{2}=-k_{n}\left|\delta z_{2}\right|-\eta_{n} \delta \dot{z}_{2}$, we can easily obtain the analytical form of $e^{\prime}$ as

$$
e^{\prime}=\exp \left(-\frac{\pi}{\sqrt{\frac{1}{1-\alpha} \frac{4 m k_{n}}{\eta_{n}^{2}}-1}}\right) .
$$

Because we adopt a nonlinear spring model in our simulation, it is difficult to obtain an analytical form of $e^{\prime}$. Thus, we 
numerically calculate $e^{\prime}$ from Eq. (33). When the duration of contact is sufficiently small, we can rewrite the contact force $F_{2}^{\prime \prime}$ by using a $\delta$ function as

$$
F_{2}^{\prime \prime}=(1-\alpha) F_{2}^{\prime}=-\left(e^{\prime}+1\right)\left(U_{i}-\dot{z}_{p}\right) \delta\left(t-t_{i}\right) .
$$

The time integral of Eq. (35) around $t_{i}$ satisfies Eq. (33). Consequently, the time evolution equation of sphere 2 at the leading order of $\alpha$ is

$$
\ddot{z}_{2}=-(1-\alpha) g+\alpha \ddot{z}_{p}+F_{2}^{\prime \prime} .
$$

Note that $\alpha /(1-\alpha) \sim \alpha$.

Now we obtain the equation of motion of sphere 2 in a form independent of the motion of sphere 1. Hereafter, the stability of sphere 2 is analyzed in the same way as for periodic bouncing of a single ball [9]. The time integral of Eq. (36) gives the relation between $V_{i}$ and $U_{i+1}$ as

$$
\begin{aligned}
U_{i+1}= & V_{i}-(1-\alpha) g\left(t_{i+1}-t_{i}\right) \\
& +\alpha A \omega\left(\cos \omega t_{i+1}-\cos \omega t_{i}\right) .
\end{aligned}
$$

Similarly, the time integral of $\dot{z}_{2}$ from $t_{i}$ to $t_{i+1}$ gives

$$
\begin{aligned}
& (1-\alpha) A\left(\sin \omega t_{i+1}-\sin \omega t_{i}\right) \\
& \quad=\left(V_{i}-\alpha A \omega \cos \omega t_{i}\right)\left(t_{i+1}-t_{i}\right)-(1-\alpha) \frac{g}{2}\left(t_{i+1}-t_{i}\right)^{2} .
\end{aligned}
$$

The relation between $V_{i+1}$ and $U_{i+1}$ can be obtained as

$$
V_{i+1}=U_{i+1}-\left(1+e^{\prime}\right)\left(U_{i+1}-A \omega \cos \omega t_{i+1}\right) .
$$

Here, we normalize the variables as $\phi_{i}=\omega t_{i}, u_{i}=U_{i} \omega / g$, $v_{i}=U_{i} \omega / g$, and $\Gamma=A \omega^{2} / g$. Thus, the governing equations are written as

$$
\begin{gathered}
\phi_{i+1}=\phi_{i}+\tau_{i} \\
u_{i+1}=v_{i}-(1-\alpha) \tau_{i}+\alpha \Gamma\left(\cos \phi_{i+1}-\cos \phi_{i}\right), \\
v_{i+1}=-e^{\prime} u_{i+1}+\left(1+e^{\prime}\right) \Gamma \cos \phi_{i+1} \\
(1-\alpha) \Gamma\left(\sin \phi_{i+1}-\sin \phi_{i}\right) \\
=\left(v_{i}-\alpha \Gamma \cos \phi_{i}\right) \tau_{i}-(1-\alpha) \frac{1}{2} \tau_{i}^{2}
\end{gathered}
$$

We assume that the height of sphere 2 is larger than the vibration amplitude [9]. Thus, we neglect the left-hand side of Eq. (41). From Eq. (41),

$$
\tau_{i}=\frac{2}{1-\alpha}\left(v_{i}-\alpha \Gamma \cos \phi_{i}\right)
$$

This system is the mapping of $\phi_{i}$ and $v_{i}$. The periodic collision with $\tau_{i}=2 \pi, \phi=\phi_{i+1}=\phi_{i}$, and $v=v_{i+1}=v_{i}$ gives a fixed point as

$$
\begin{gathered}
v=\pi(1-\alpha)+\alpha \Gamma \cos \phi, \\
\Gamma \cos \phi=\pi \frac{1-e^{\prime}}{1+e^{\prime}} .
\end{gathered}
$$

The stability of the fixed point of the system is determined by the eigenvalues of the Jacobian matrix as

$$
\begin{aligned}
\frac{\partial \phi_{i+1}}{\partial \phi_{i}}= & 1+\frac{\partial \tau_{i}}{\partial \phi_{i}}, \\
\frac{\partial \phi_{i+1}}{\partial v_{i}}= & \frac{\partial \tau_{i}}{\partial v_{i}}, \\
\frac{\partial v_{i+1}}{\partial \phi_{i}}= & -\left\{\alpha+(1-\alpha)\left(1+e^{\prime}\right)\right\} \Gamma \sin \left(\phi_{i}+\tau_{i}\right) \frac{\partial \phi_{i+1}}{\partial \phi_{i}} \\
& +\alpha e^{\prime} \Gamma \sin \left(\phi_{i}\right), \\
\frac{\partial v_{i+1}}{\partial v_{i}}= & e^{\prime}-\left\{\alpha+(1-\alpha)\left(1+e^{\prime}\right)\right\} \Gamma \sin \left(\phi_{i}+\tau_{i}\right) \frac{\partial \phi_{i+1}}{\partial v_{i}} .
\end{aligned}
$$

Here, we consider the stability of the fixed point $\tau_{i}=2 \pi$. By taking the derivative of Eq. (41) with Eq. (43), we obtain

$$
\begin{gathered}
\frac{\partial \tau_{i}}{\partial v_{i}}=\frac{2}{1-\alpha}, \\
\frac{\partial \tau_{i}}{\partial \phi_{i}}=\alpha \Gamma \sin \phi \frac{\partial \tau_{i}}{\partial v_{i}} .
\end{gathered}
$$

Then, the trace and determinant of the Jacobian matrix are written as

$$
\begin{gathered}
\frac{\partial \phi_{i+1}}{\partial \phi_{i}}+\frac{\partial v_{i+1}}{\partial v_{i}}=\left(1+e^{\prime}\right)(1-2 \Gamma \sin \phi), \\
\left(\frac{\partial \phi_{i+1}}{\partial \phi_{i}} \frac{\partial v_{i+1}}{\partial v_{i}}-\frac{\partial \phi_{i+1}}{\partial v_{i}} \frac{\partial v_{i+1}}{\partial \phi_{i}}\right)=e^{\prime} .
\end{gathered}
$$

Therefore, the eigenvalues $\lambda_{ \pm}$of the Jacobian matrix are

$$
\lambda_{ \pm}=\frac{1}{2}\left(1+e^{\prime}-\gamma \pm \sqrt{\left(1+e^{\prime}-\gamma\right)^{2}-4 e^{\prime}}\right),
$$

where $\gamma=2\left(1+e^{\prime}\right) \Gamma \sin \phi$. If we assume $1-e^{\prime}+\gamma>0$, the condition $\lambda_{+}<1$ leads to $\gamma>0 . \gamma>0$ ensures $1-e^{\prime}+\gamma>$ 0 . Then, we obtain the conditions as

$$
\sin \phi>0 \text {. }
$$

This, in conjunction with Eq. (44), gives the lower limit of $\Gamma$ as

$$
\Gamma>\Gamma_{c}=\pi \frac{1-e^{\prime}}{1+e^{\prime}} .
$$

Because $e^{\prime}$ is a function of $\alpha$, the effect of the shape of the dimer is taken into account. Nevertheless, if the restitution coefficient of the upper sphere is defined as Eq. (33), $\Gamma_{c}$ has functional form identical to that of a single bouncing ball with an ordinary restitution coefficient $e$ [9]. As a result, as shown in Fig. 10, we confirmed that the bifurcation points $\Gamma_{c}$ obtained in the numerical simulations show excellent agreement with the theoretical curve of Eq. (49). With a decrease in $e^{\prime}$, the experimental $\Gamma_{c}$ deviates from the numerical and analytical results. When $e^{\prime}$ is smaller, the contact duration becomes longer, and the discoid shape of the ends is expected to have a nonnegligible effect. This reveals for the first time that mode bifurcation of a dumbbell from directional to random motion can be well-interpreted analytically irrespective of chirality by adopting a simple mechanical model. 


\section{CONCLUSION}

We observed cascade of different modes on twodimensional motions by introducing chiral asymmetry into a dumbbell particle under vertical vibration. The SR mode shows spinning motion accompanied by random behavior of the centroid, whereas the $\mathrm{O}$ mode shows spinning motion accompanied by directional motion. In addition, the SR and $\mathrm{O}$ modes switched in a subcritical manner as a function of $\Gamma$. In numerical simulations, a simple three-dimensional model particle reproduced the SR and O modes. It was also shown that the angular velocity of the spinning motion that originates in the chirality is determined by $\sin \left(\beta^{\prime} / 2\right)$. A simple mechanical model was considered to obtain the lower limit of $\Gamma$ for the directional motion of the dumbbell irrespective of chirality. The estimated critical acceleration $\Gamma_{c}$ agreed well with the numerical results. The condition for periodic collision of the upper sphere appeared to have functional form identical to that of a single bouncing ball. An analysis of the bifurcation from the $\mathrm{O}$ mode to the rolling mode is in progress.

\section{ACKNOWLEDGMENTS}

This work was partially supported by Ministry of Education, Culture, Sports, Science and Technology (MEXT)/Japan Society for the Promotion of Science (JSPS) KAKENHI through Grants No. 23240044, No. 25103012, No. 26707020, and No. 12J40224. This work was also supported by the JSPS Core-to-Core Program "Nonequilibrium dynamics of soft matter and information."

\section{APPENDIX: THE EFFECT OF BOUNCING OF THE LOWER SPHERE}

In this appendix, we consider the flight of sphere 1 . When sphere 2 collides with the plate, sphere 1 leaves the bottom plate and bounces on it a few times before resting on the bottom plate again. We define $t_{i}$ as the sequence of collision times of sphere 2 . Then, we assume that sphere 1 undergoes the $j$ th collision with the bottom plate at $t=t_{i}+s_{j}$. Here, we define the normalized $j$ th collision time, preimpact velocity, and postimpact velocity of sphere 1 as $\psi_{j}, u_{1, j}$, and $v_{1, j}$, respectively. From Eqs. (28) and (35), the mappings of sphere 1 are

$$
\begin{gathered}
\psi_{j+1}=\psi_{j}+\chi_{j}, \\
u_{1, j+1}=-\chi_{j}+v_{1, j}, \\
v_{1, j+1}=-e_{j+1} u_{1, j+1}+\left(e_{j+1}+1\right) \Gamma \cos \psi_{j+1}, \\
\Gamma\left(\sin \psi_{j+1}-\sin \psi_{j}\right)=-v_{1, j} \chi_{j}+\frac{1}{2} \chi_{j}^{2},
\end{gathered}
$$

where $e_{j}$ is the restitution coefficient of the $j$ th collision of sphere 1 . The initial conditions are

$$
\begin{gathered}
v_{1,0}=\Gamma \cos \phi_{i}-\alpha\left(e^{\prime}+1\right)\left(u_{i}-\Gamma \cos \phi_{i}\right), \\
\psi_{0}=0 .
\end{gathered}
$$

Here, we assume that sphere 1 rests on the bottom plate after the $n$th collision. Then, the normal force $F_{1}^{\prime}$ acting on sphere 1 is modified as

$$
\begin{gathered}
F_{1}^{\prime}=-\sum_{j=1}^{n}\left(1+e_{j}\right)\left(u_{1, j}-\dot{z}_{p}\left(\psi_{j}\right)\right) \delta\left(\phi-\psi_{j}\right), \\
\left(\phi_{i}<\phi<\phi_{i}+\psi_{n}\right) \\
F_{1}^{\prime}=\frac{\Gamma \sin \phi+1}{1-\alpha}, \quad\left(\phi_{i}+\psi_{n}<\phi<\phi_{i+1}\right) .
\end{gathered}
$$

By using the step function $\theta, F_{1}^{\prime}$ is written as

$$
\begin{gathered}
F_{1}^{\prime}=\frac{\Gamma \sin \phi+1}{1-\alpha}+F^{\prime} \\
F^{\prime}=-\theta\left(\phi_{i}<\phi<\phi_{i}+\psi_{n}\right) \frac{\Gamma \sin \phi+1}{1-\alpha} \\
-\sum_{j=1}^{n}\left(1+e_{j}\right)\left[u_{1, j}-\dot{z}_{p}\left(\psi_{j}\right)\right] \delta\left(\phi-\psi_{j}\right) .
\end{gathered}
$$

If Eqs. (35) and (A9) are substituted into Eq. (29), the equation of sphere 2 is

$$
\ddot{z}_{2}=-\frac{1-2 \alpha}{1-\alpha} g+\frac{\alpha}{1-\alpha} \ddot{z}_{p}+F_{2}^{\prime \prime}+\alpha F^{\prime} .
$$

Thus, $\alpha F^{\prime}$ represents the deviation due to the flight and collision of sphere 1 . Here, we treat $\alpha F^{\prime}$ as a perturbation. Then, we calculate $F^{\prime}$ from Eqs. (A1)-(A6) with the nonperturbed solution $\Gamma=\Gamma_{c}, \phi_{i}=0$, and $\tau_{i}=2 \pi$. Subsequently, $F^{\prime}$ becomes constant, and the lower limit of $\Gamma$ is easily calculated:

$$
\begin{gathered}
\Gamma>\Gamma_{c}+\delta \Gamma, \\
\delta \Gamma=\alpha\left(\frac{J}{2 \pi}+\frac{e^{\prime}}{1+e^{\prime}} I\right), \\
I=-\frac{1}{1-\alpha}\left(\Gamma_{c} \cos \psi_{n}-\Gamma_{c}+\psi_{n}\right) \\
-\sum_{j=1}^{n}\left(1+e_{j}\right)\left(u_{1, j}-\Gamma_{c} \cos \psi_{j}\right), \\
J=-\frac{1}{1-\alpha}\left(\Gamma_{c} \sin \psi_{n}-\Gamma_{c} \psi_{n}+\frac{1}{2} \psi_{n}^{2}\right) \\
-I\left(2 \pi-\psi_{n}\right) \\
-2 \pi \sum_{j=1}^{n}\left(1+e_{j}\right)\left(u_{1, j}-\Gamma_{c} \cos \psi_{j}\right) \\
+\sum_{j=1}^{n}\left(1+e_{j}\right)\left(u_{1, j}-\Gamma_{c} \cos \psi_{j}\right) \psi_{j},
\end{gathered}
$$

where the initial conditions are $v_{1,0}=\Gamma_{c}+2 \pi \alpha$ and $\psi_{0}=0$. For simplicity, we assume $e_{j}=e^{\prime}$ and calculate $\delta \Gamma$ numerically. Except for $e \sim 1$, we confirm that $\delta \Gamma / \Gamma_{c}$ is in the order of 0.01 . 
For $\Gamma_{c}>1$, sphere 1 leaves the plate again at $\psi=\psi_{d}=$ $\sin ^{-1}\left(1 / \Gamma_{c}\right)$. We can calculate the effect of this flight from Eqs. (A12)-(A15) with $v_{1,0}=\Gamma_{c} \cos \psi_{d}$ and $\psi_{0}=\psi_{d}$. In this case, the trajectory of sphere 1 could be complex. However, the flight of sphere 1 changes the lower limit of $\Gamma$ by $\sim 1 \%$.
[1] H. Chaté, F. Ginelli, and R. Montagne, Simple model for active nematics: Quasi-long-range order and giant fluctuations, Phys. Rev. Lett. 96, 180602 (2006).

[2] V. Narayan, S. Ramaswamy, and N. Menon, Long-lived giant number fluctuations in a swarming granular nematic, Science 317, 105 (2007).

[3] A. Kudrolli, G. Lumay, D. Volfson, and L. S. Tsimring, Swarming and swirling in self-propelled polar granular rods, Phys. Rev. Lett. 100, 058001 (2008).

[4] J. Deseigne, O. Dauchot, and H. Chaté, Collective motion of vibrated polar disks, Phys. Rev. Lett. 105, 098001 (2010).

[5] L. A. Wood and K. P. Byrne, Analysis of a random repeated impact process, J. Sound Vib. 78, 329 (1981).

[6] P. J. Holmes, The dynamics of repeated impacts with a sinusoidally vibrating table, J. Sound Vib. 84, 173 (1982).

[7] C. N. Bapat, S. Sankar, and N. Popplewell, Repeated impacts on a sinusoidally vibrating table reappraised, J. Sound Vib. 108, 99 (1986).

[8] N. B. Tufillaro and A. M. Albano, Chaotic dynamics of a bouncing ball, Am. J. Phys. 54, 939 (1986).
[9] J. J. Barroso, M. V. Carneiro, and E. E. N. Macau, Bouncing ball problem: Stability of the periodic modes, Phys. Rev. E 79, 026206 (2009).

[10] S. Dorbolo, D. Volfson, L. Tsimring, and A. Kudrolli, Dynamics of a Bouncing Dimer, Phys. Rev. Lett. 95, 044101 (2005).

[11] D. Yamada, T. Hondou, and M. Sano, Coherent dynamics of an asymmetric particle in a vertically vibrating bed, Phys. Rev. E 67, 040301 (2003).

[12] H. S. Wright, Michael R. Swift, and P. J. King, Stochastic dynamics of a rod bouncing upon a vibrating surface, Phys. Rev. E 74, 061309 (2006).

[13] J. Wang, C. Liu, Y.-B. Jia, and D. Ma, Ratchet rotation of $a_{3}$ D dimer on a vibrating plate, Eur. Phys. J. E 37, 1 (2014).

[14] See Supplemental Material at http://link.aps.org/supplemental/ 10.1103/PhysRevE.91.052905 for movies of dumbbell motion.

[15] K. Iwashita and M. Oda, Rolling resistance at contacts in simulation of shear band development by DEM, J. Eng. Mech. 124, 285 (1998).

[16] H. J. Herrmann and S. Luding, Modeling granular media on the computer, Continuum Mech. Thermodynam. 10, 189 (1998). 